Forum $2020 \cdot 35: 406-407$

https://doi.org/10.1007/s12312-020-00844-6

Online publiziert: 16 . September 2020

(c) Springer Medizin Verlag GmbH, ein Teil von Springer Nature 2020

\title{
Christina Große-Thie
}

Medizinische Klinik III, Hämatologie, Onkologie und Palliativmedizin, Universitätsmedizin Rostock, Rostock, Deutschland

\section{Erratum zu: Implikationen moderner onkologischer Therapien für die Palliativversorgung}

\section{Erratum zu:}

Forum 2020

https://doi.org/10.1007/s12312-02000774-3

In der • Tab. 1 „Übersicht zugelassene Immun-Checkpoint-Inhibitoren in Deutschland“, Zeile 7 „Wirkstoff: Cemiplimab" hat sich leider ein Fehler eingeschlichen.

Bei Cemiplimab handelt es sich um einen PD-1-Antikörper und NICHT, wie versehentlich angegeben, um einen $\mathrm{PD}$ L1-Antikörper.
Wir bitten, diesen Fehler zu entschuldigen.

Nachstehend nochmals die Tabelle in korrigierter Form.

\section{Korrespondenzadresse}

Dr. Christina Große-Thie

Medizinische Klinik III, Hämatologie, Onkologie und Palliativmedizin, Universitätsmedizin Rostock

Ernst-Heydemann-Str. 6, 18057 Rostock, Deutschland christina.grosse-thie@med.uni-rostock.de

\begin{tabular}{|c|c|c|c|}
\hline Wirkstoff & Handelsname & Wirkprinzip & Zugelassene Indikationen \\
\hline Ipilimumab & Yervoy ${ }^{\circledR}$ & CTLA-4-Antikörper & Malignes Melanom, Nierenzellkarzinom \\
\hline Nivolumab & Opdivo ${ }^{\circledR}$ & PD1-Antikörper & $\begin{array}{l}\text { Malignes Melanom, NSCLC, SCCHN, } \\
\text { Urothelkarzinom, M. Hodgkin, Nieren- } \\
\text { zellkarzinom }\end{array}$ \\
\hline Pembrolizumab & Keytruda $^{\circledR}$ & PD1-Antikörper & $\begin{array}{l}\text { Malignes Melanom, HNSCC, NSCLC, } \\
\text { Urothelkarzinom, Nierenzellkarzinom, } \\
\text { Morbus Hodgkin }\end{array}$ \\
\hline Atezolizumab & Tecentriq $^{\circledR}$ & PD-L1-Antikörper & NSCLC, Urothelkarzinom, SCLC, TNBC \\
\hline Durvalumab & $\operatorname{Imfinzi}^{\circledR}$ & PD-L1-Antikörper & NSCLC (adjuvant) \\
\hline Avelumab & Bavenico $^{\circledR}$ & PD-L1-Antikörper & $\begin{array}{l}\text { Merkelzellkarzinom, Nierenzellkarzi- } \\
\text { nom }\end{array}$ \\
\hline Cemiplimab & Libtayo ${ }^{\circledR}$ & PD-1-Antikörper & Spinaliom \\
\hline
\end{tabular}

Die Online-Version des Originalartikels ist unter https://doi.org/10.1007/s12312-020-00774-3 zufinden. 
Hier steht eine Anzeige.

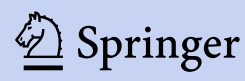

